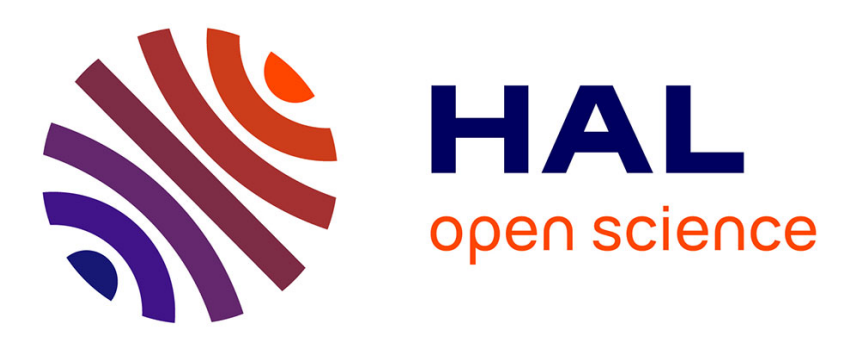

\title{
Resilient, Decentralized V2V Online Stop-Free Strategy in a Complex Roundabout
}

\author{
Marie-Ange Lèbre, Frédéric Le Mouël, Eric Ménard
}

\section{To cite this version:}

Marie-Ange Lèbre, Frédéric Le Mouël, Eric Ménard. Resilient, Decentralized V2V Online Stop-Free Strategy in a Complex Roundabout. Proceedings of the IEEE 83rd Vehicular Technology Conference (VTC Spring 2016), May 2016, Nanjing, China. 10.1109/VTCSpring.2016.7504449 . hal-01346423

\section{HAL Id: hal-01346423 \\ https://hal.inria.fr/hal-01346423}

Submitted on 18 Jul 2016

HAL is a multi-disciplinary open access archive for the deposit and dissemination of scientific research documents, whether they are published or not. The documents may come from teaching and research institutions in France or abroad, or from public or private research centers.
L'archive ouverte pluridisciplinaire HAL, est destinée au dépôt et à la diffusion de documents scientifiques de niveau recherche, publiés ou non, émanant des établissements d'enseignement et de recherche français ou étrangers, des laboratoires publics ou privés. 


\title{
Resilient, Decentralized V2V Online Stop-free Strategy in a Complex Roundabout
}

\author{
Marie-Ange Lèbre* ${ }^{* \dagger}$, Frédéric Le Mouël* Eric Ménard ${ }^{\dagger}$ \\ * University of Lyon, INSA-Lyon, CITI-INRIA, F-69621 Villeurbanne, France \\ Email: $\{$ marie-ange.lebre, frederic.le-mouel $\}$ @insa-lyon.fr \\ $\dagger$ VALEO, Advanced Technology Developement, F-94000 Creteil, France \\ Email: \{marie-ange.lebre, eric.menard\} @valeo.com
}

\begin{abstract}
Road intersections are considered to be bottlenecks for urban transportation whose impacts are longer travel times and wasted human resources. In this paper we focus on vehicle to vehicle communications (V2V) that allow exchanging data between vehicles. Considering that vehicles are controlled by drivers (not autonomous), we do not pretend to take control of them, nor is the goal to avoid collision or improve safety, as is often done elsewhere. By eliminating the potential overlaps of vehicular trajectories coming from all opposing directions at an intersection, our aim is to demonstrate the potential of communication between vehicles in a complex roundabout and test the connexion strength of that network. We test it on a synthetic trace that reproduces a real traffic flow at a roundabout in Creteil (France).
\end{abstract}

Keywords-synthetic trace, real data, V2V, packet loss, radius of communication, roundabout, traffic lights.

\section{INTRODUCTION}

Road intersections are currently managed by stops or traffic lights for improve the safety. There is a growing interest in the efficiency and safety of intersections. The delays due to stop signs and red traffic lights significantly increase travel times. Intelligent transport systems allow imagining solutions for crossing an intersection or a roundabout more efficiently. For instance, some models are based on a reservation process with the First Come First Serve protocol, whereby vehicles send a request to the intersection to cross it [1]. Mourad Ahmane et al. [2] proposed the use of a Petri network to find the optimal passing sequence with communication between vehicles via a server.

The system can be also decentralized, for instance, in the case where a vehicle does not have right of way, it can send a request to other vehicles to negotiate it. The vehicle may be obliged to stop at an intersection [3]. Javier Alonso et al. [4] proposed a cooperative maneuver, by adjusting the speed, between vehicles equipped with sensors, whether driven manually or autonomously. A collision avoidance scheme was presented by Gabriel Rodrigues et al [5], with decentralized coordination between vehicles based on the freedom degrees that each vehicle has available to avoid collision.

In this paper we present a decentralized V2V fluidity model by eliminating the potential overlaps of vehicular trajectories. In the first section we present the roundabout dataset. In the second section we describe our model and in the last section we test its robustness with respect to network performance.

\section{THE EUROPARC ROUNDABOUT DATASET}

The vehicular mobility trace used in our paper focuses on a roundabout located in Creteil in France in the working area known as Europarc. The paper [6] (data available on the website) describes the traffic mobility on this large roundabout with the highest level of realism and the real traffic light system is included. It provides details on the characteristics of the different components and the process used to generate the mobility trace.

\section{A. Vehicular mobility}

The microscopic mobility of vehicles is simulated with the Simulator of Urban MObility (SUMO 0.22). The models implemented by SUMO for moving vehicles is the Krauss car following model. It integrates the safety distance between cars and allows setting acceleration, deceleration and driver behavior individually. The model employed for changing lanes is Krajzewicz's lane-changing model which allows regulating and distributing the traffic in the case of multiple lanes and it can make overtaking decisions. These models have been widely validated in the transportation research community.

\section{B. Traffic data}

The traffic information used on the roundabout is taken from real observations of vehicle flows. An O/D matrix faithfully mimics the daily movements of vehicles in the area for a period of 4 hours : $7.15 \mathrm{AM}$ to $9.15 \mathrm{AM}$ and 5.00 PM to 7.00 PM on tuesday in a working week in September. This represents around 5000 trips in the morning and, on average, the same number in the evening. The roundabout

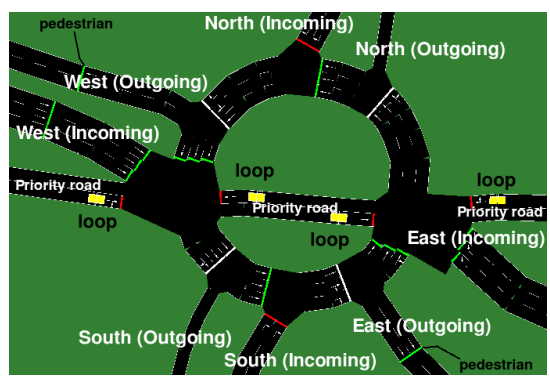

Fig. 1: Outgoing and incoming edges

is composed of 6 entries and 6 exits, 2 entries and 2 exits are exclusively reserved for buses. The road for buses is indicated in figure 1 by visible induction loops. Other induction loops -not indicated- exist on external roads to measure rates of occupancy. 15 traffic lights are included in the map: 12 are located inside it to regulate traffic ; 3 are for pedestrians outside and work even if there are no pedestrians ; 4 of the 12 traffic lights are dedicated for buses. In addition, 4 white 
lanes inside are intersections without traffic lights -non-stop intersections- that indicate the exits of the roundabout.

\section{V2V FLUIDITY SYSTEM}

\section{A. Detecting and Resolving a Conflict}

In our model, traffic lights are off, except those for pedestrians. The system provides a recommended speed for entering the roundabout with fluidity. Time in our model is a discrete value expressed in seconds and the distances are expressed in meters. Using $\mathrm{V} 2 \mathrm{~V}$ communication the vehicles send "Time Lists" (TL) to other vehicles which allow them to detect a conflict and resolve it as a function of priority rules. The steps presented in the algorithm 1 are applicable to all vehicles. As crossing a roundabout at $13.89 \mathrm{~m} / \mathrm{s}$, a high speed

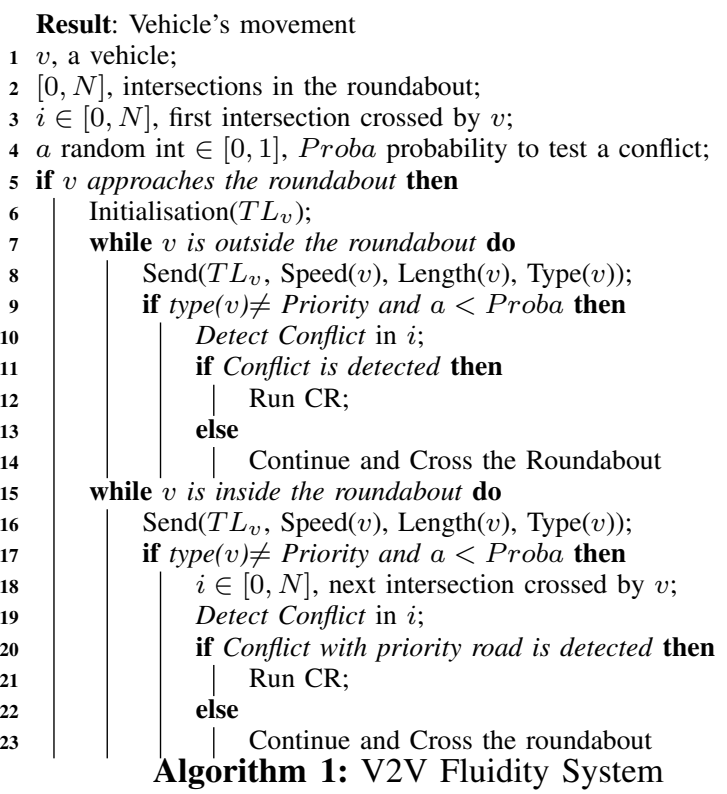

for this kind of intersection, can be difficult for a driver, we limit the speed in the roundabout to $8.5 \mathrm{~m} / \mathrm{s}$. Considering the presentation of our physical context in the previous section, the roundabout has $N=4$ intersections that we denote $w$ for West, $s$ for South, $e$ for East and $n$ for North, $[w, s, e, n]$ is the list of the four intersections in the roundabout. Each vehicle $v$ has a "Time List", $T L_{v}$, which describes the passage times at the different intersection. In our context: $T L_{v}$ is equal to $\left[t_{w}, t_{s}, t_{e}, t_{n}\right]$. When the vehicle arrives near the roundabout, it initializes its list $T L_{v}$ (line 2). This initialization uses the current speed of the vehicle and its position. By using Euclidean distance, the vehicle has an approximation of its arrival time at each intersection $[w, s, e, n]$. This assumes that the speed of the vehicle is constant until its arrival in the roundabout. Detect Conflict on line 6 runs on all the information received by the vehicle, which is broadcast by the surrounding vehicles. This function uses the $T L_{y}$ of senders $y$ and $T L_{v}$ of vehicle $v$. Detect Conflict compares the lists and determines if there is any shorter time interval between the common cells along the trajectories of the vehicles in the roundabout at cells $w, s, e$ and $n$ (see figure 2 (A)). This time interval corresponds to the space necessary for a vehicle to be inserted after or before another vehicle $y$ (around 2.2 seconds [7], see the subsection III-C1). If a potential conflict is detected by Detect Conflict, the algorithm runs the Conflict
Resolution : CR, which consists of an acceleration if it's possible (maximum allowed speed) or a deceleration and an update of the $T L_{v}$. If several conflicts are detected, and if the vehicle is not able to accelerate enough (maximum speed), CR chooses the minimum deceleration between all the conflicts. Finally our algorithm is probabilistic, the proba allows to detect or not a conflict.

Figure 2 (A) illustrates a scenario where there are common

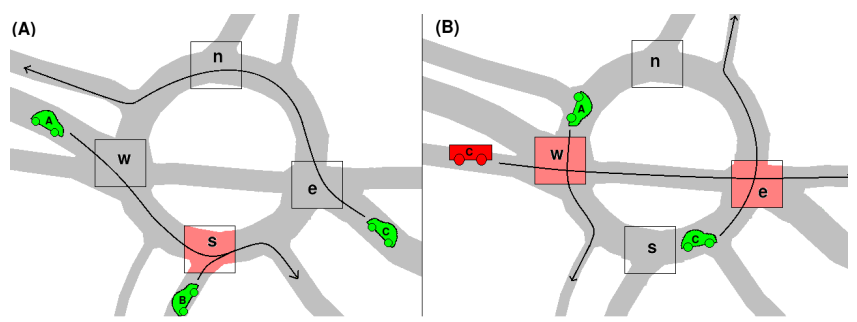

Fig. 2: (A) Example in which conflicts occur, (B) Example in which priority conflicts appear : Bus $\mathrm{C}$ is in the priority road, vehicles A and $\mathrm{B}$ detect conflict with this bus and have to run $\mathrm{CR}$.

cells along the trips of vehicles B and A. A collision can occur in cell $s$. As we apply the First Come, First Serve protocol, vehicle $A$ will be the first vehicle in the roundabout, therefore vehicle B will detect a collision and run Conflict Resolution. On the other hand, vehicle $\mathrm{C}$ has no detected conflict and continues its trip by crossing the roundabout.

\section{B. Priority rules}

Vehicles are assumed to follow the First-Come, FirstServe (FCFS) protocol in which the vehicle with the shortest arrival time at the roundabout has the highest priority. In fact, vehicles which enter the roundabout have priority over the vehicles outside it. Also, buses or any emergency vehicle on the priority road have priority over the vehicles driving inside the roundabout. The second part of the algorithm (line 11) processes this situation. Vehicles inside the roundabout decelerate when the priority vehicles arrive. This scenario is illustrated in figure 2 (B). To sum up, the priority rules in decreasing order are: 1) vehicle on the priority road, 2) vehicle inside the roundabout, 3) vehicle closest to the roundabout, 4) vehicle outside the roundabout.

\section{Constraints}

1) Safety distance: In our model, the Time Lists change as the vehicle makes progress outside the roundabout and resolves conflicts. The updated TL contains new times for each cell if a conflict has been processed. This can lead to an infinite loop, with acceleration and deceleration until the vehicle arrives at the roundabout. However, in our V2V model, as vehicles never stop the safety distance is respected and it allows to resolve conflicts : the phenomenon is absorbed. Then, the functionality of a roundabout is directly related to the ability of entering drivers to select an appropriate gap within the circulating traffic stream. The critical gap refers to a specific gap size which a driver would accept if it were larger, and would reject it if it were smaller. The authors of [7] studied the critical gap of roundabouts and determined an accurate and reliable value. The value is on average 12.80 meters in space and 2.2 seconds in time. SUMO includes the safe distance between cars in accordance with Krauss model. As the vehicles in the roundabout have priority, they follow the speed limit set to $8.5 \mathrm{~m} / \mathrm{s}$, so the safe distance is on average 
20 meters in space and $2.5 \mathrm{~s}$ in time. The result of this distance is that a vehicle that enters the roundabout has time to achieve its insertion.

2) Geographic localisation: Our system involves geographic knowledge of the roundabout. Each vehicle knows its geographic position and the geographic positions of each intersections. Therefore a global positioning system knowledge is needed. We assume that all the vehicles have access to this information. This assumption is realistic because the units available on the market for $\mathrm{V} 2 \mathrm{~V}$ communication provide an embedded GPS. However if the localization device is not available, solutions exist such as in [8] in which they propose a cooperative vehicle positioning system that provides accurate positions in a situation where some vehicles have a GPS device. In our model, each vehicle bases its safety distance on a positioning error. This increases the buffer distance between vehicles to give them the capacity to slow down when the leader vehicle has to slow down suddenly. When the vehicle computes its Time List, we add 1 second, representing a margin ranging from $5 \mathrm{~m}$ to $14 \mathrm{~m}$ (depending on the speed of $20-50 \mathrm{~km} / \mathrm{h}$ outside the roundabout).

\section{Simulations AND Results}

\section{A. Impact of the V2V Fluidity Model}

We first considered that drivers follow the V2V system and all vehicles had a communication device. First of all, we focused on the occupancy rate of the following roads: West, South, East and North (see figure 11). Two traffic lights were reserved for pedestrians; we considered them in the V2V Fluidity Model. We focused our simulations on the dataset for the evening from 5 PM to 7 PM, representing 5000 trips.

As can be seen in figure 3, the occupancy rates for the outgoing edges are similar in the two situations. Then, for the incoming edges the results are better with the V2V model than in the real scenario with traffic lights. Vehicles in our model never stopped moving and no accumulation of vehicles occurred in the incoming roads. Moreover, the vehicles never
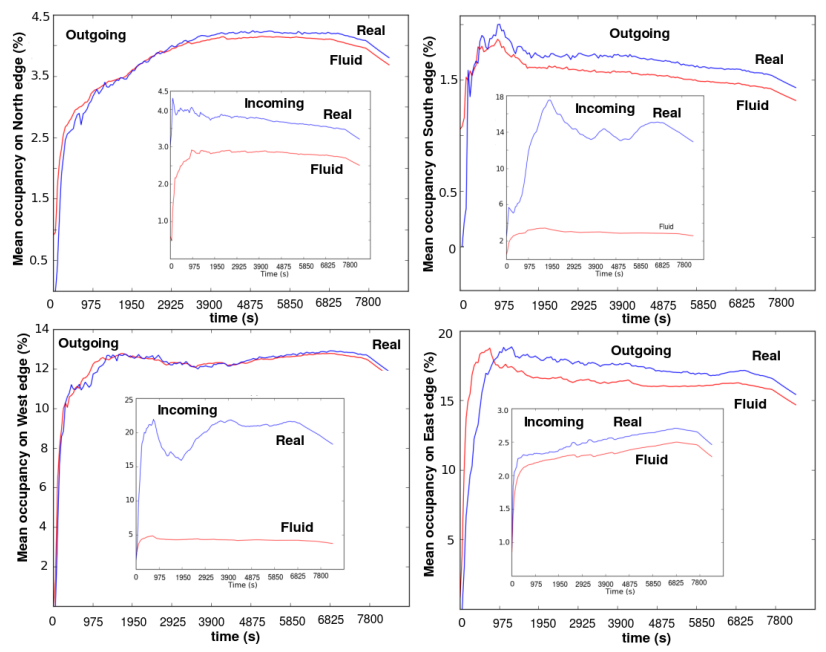

Fig. 3: Mean occupancy at the outgoing edges and the incoming edges

stop so obviously the waiting time in figure 4 is reduced. The mean difference between the mean waiting time between the two scenarios is around 30 seconds. This value corresponds, on average, to one red traffic light duration in the roundabout. It means that on average a vehicle waits at one traffic light in the roundabout. This waiting time affected travel time with an improvement of around 57 seconds in the evening. This appears negligible, but can be significant when taking the entire travel time of the vehicle into account. The time taken
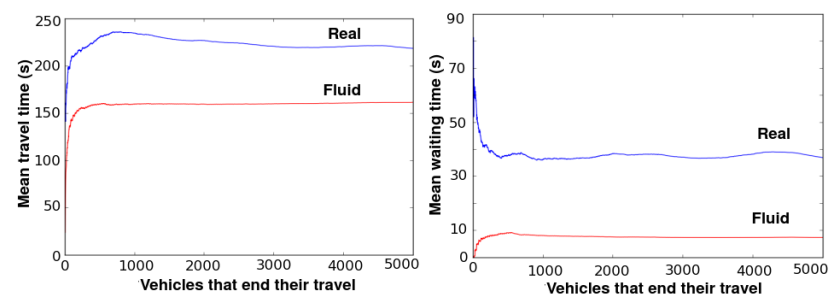

Fig. 4: Mean travel time and mean waiting time

to cross the roundabout in the real scenario was 29.5 seconds on average and 11.8 seconds with our model. Then, if we take the incoming edges into account, when vehicles were able to receive information from other vehicles, the average time to cross the roundabout was 86.5 seconds in the real case and 43.5 seconds with our model. Therefore the gain was achieved before the crossing of the roundabout.

The figure 5 represents the number of vehicles according to their travel times and waiting times on the whole map with the real case and our V2V model. The average travel time in our model is 162.02 seconds against $219 \mathrm{~s}$ in real case, and $278 \mathrm{~s}$ is the maximum value against $472 s$. The average waiting time is $7.51 \mathrm{~s}$ against $37 \mathrm{~s}$ and the maximum is $56 \mathrm{~s}$ against $185 \mathrm{~s}$. Our model outperforms the real case and moreover the distribution is quite fair in waiting time and travel time.

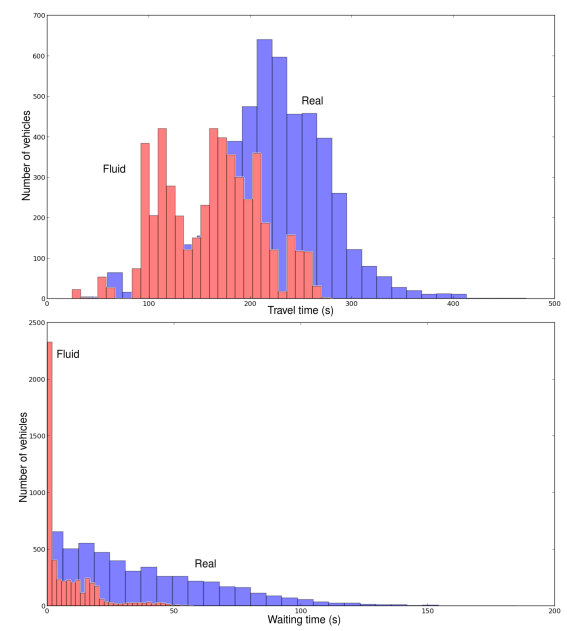

Fig. 5: Histogram of travel time and waiting time

\section{B. Comparison with two other models}

We compare with a Reservation model and a Negotiation model. The first one is centralized with an intersection management agent based on a reservation table model with an optimal scheduling as in the paper [9]. The second one is decentralized [10], the model is based on negotiation of the right of way. Vehicle close to the vehicle in front obtains a right of way as long as the vehicle in front has it, and if a vehicle has not right of way it negotiate with vehicles in 
conflict and the closest one obtains the right of way.

Our V2V Fluidity Model and the Negotiation model have similar results (figure 6) with a difference in duration around $2,10 \%$, and around $9.84 \%$ with the centralized solution. Then for waiting time, V2V Fluidity Model is better of $0.3 \%$ than the Negotiation model and is close to the centralized solution with a difference of $2.5 \%$.

In figure 8 , we summary the average time spent by vehicles

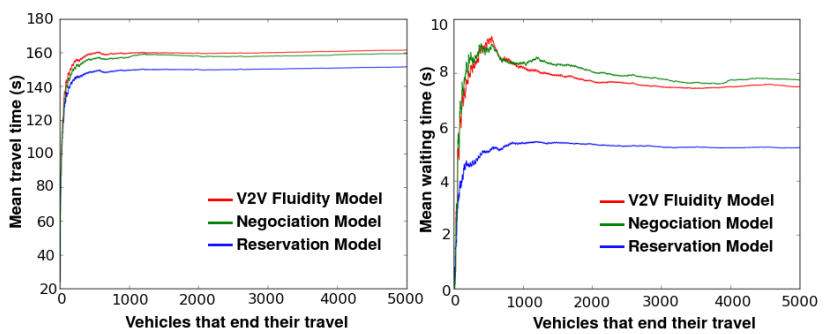

Fig. 6: Comparison with a centralized (Reservation) and a decentralized system (Negotiation)

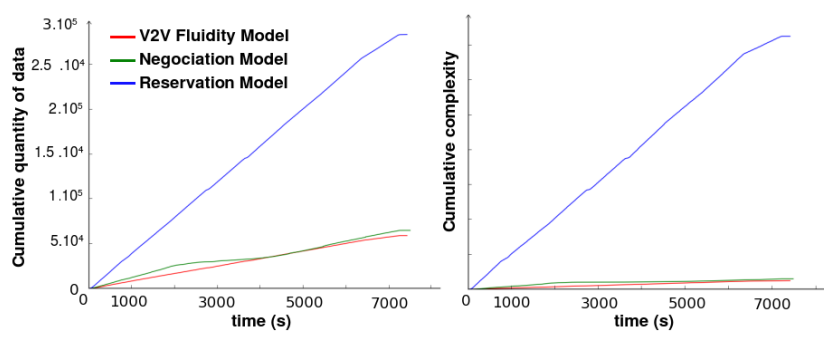

Fig. 7: Cumulative quantity of data and complexity with the three models

\begin{tabular}{|c|c|c|c|}
\hline Model & V2V Model & Negotiation & Reservation \\
\hline Time spent in the RA & 11.80 & 12.97 & 7.70 \\
Time to cross the RA & 43.5 & 42.10 & 34.75 \\
\hline
\end{tabular}

Fig. 8: Time spent in the RoundAbout (RA) and for crossing it according to the different models

in the RoundAbout (RA) and the time for crossing it ( it begins when vehicles are able to receive information from other vehicles). We win in average $1.2 \mathrm{~s}$ in the roundabout but lose $1.4 \mathrm{~s}$ for crossing it against the negotiation model but we are close the Reservation model. Unlike the negotiation model, in our model, vehicles don't depend of vehicles front of it to obtain the right of way, an individual control gives similar results. Finally in figure 7, we notice that the quantity of data and the complexity of our fluidity model is better than the two others.
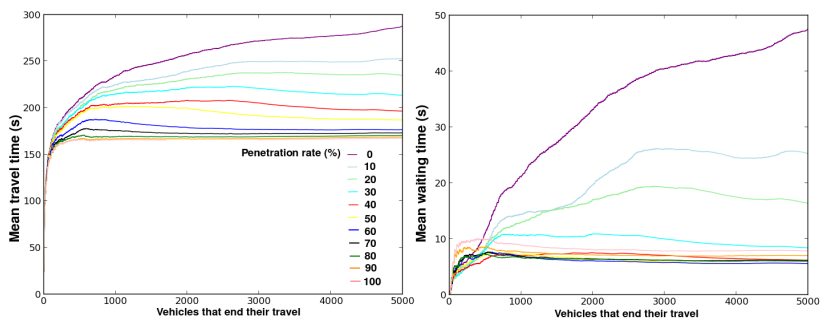

Fig. 9: Mean travel time and waiting time with different equipment rates

\section{Robustness: Rate of equipment}

We test the robustness of our model according to the equipment rate in $\mathrm{V} 2 \mathrm{~V}$ devices. In order to introduce human behavior, the erratic driving parameter in the model of Krauss is set to 0.8 , which means that drivers drive their vehicles more nervously (peak hour). Our system is local, therefore the rate of penetration has an impact on the data flow and on the performance of the system. This test can be assimilated to a test on the cooperation of the drivers with the system, on their capacity to follow the recommendation. Figure 9 shows the results in mean travel time and waiting time. We notice that they are significant with an equipment rate around $50 \%$. Equipped vehicles have an impact on non-equipped vehicles.

\section{Robustness: Density}

Vehicular density in the roundabout between 5PM and 7PM is not very heavy and traffic lights under real conditions add situations of congestion. The geographic capacity of the roundabout can absorb the real traffic flow without traffic lights. Therefore using real data, we add trips by conforming to the flow distribution of the real data. Figure 10 shows the mean travel time and the mean waiting time for different densities. Results of our model are stable for all densities. For 7900 and 8500 trips the roundabout with traffic lights is not able to evacuate all vehicles and the bottleneck appears in the roundabout. Overall, our system supports the density and the system explodes in travel time and waiting time between 6900 and 7900 vehicles in the simulation with traffic lights. The traffic light system is not efficient in this situation. We must note that the travel time and the waiting time, decrease at the end of the simulation in the real system, because the arrival law is null.
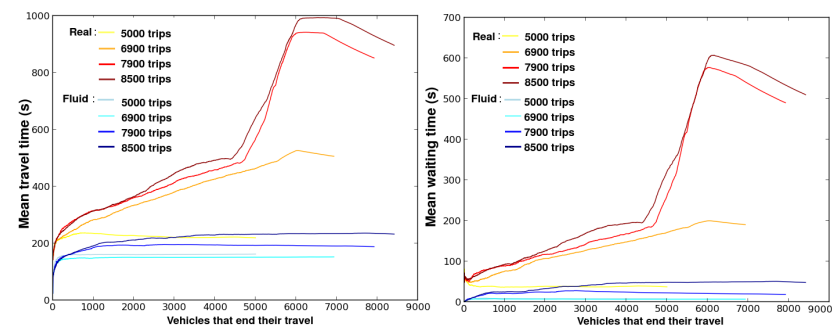

Fig. 10: Mean travel time and waiting time with different densities

\section{E. Robustness: Packet Loss}

We now evaluate the impact of packet loss. The figure 11 (A) shows results with real density. We don't represent $100 \%$ of packet loss, as it corresponds to the situation where no vehicle is equipped (see section IV-C). More packet are lost, more travel time is high, with a difference of $15 \%(27 s)$ between $80 \%$ and $0 \%$ of packet loss. But the difference is not significant. However there is an important gap between the situation where no vehicle is equipped in section IV-C ( $100 \%$ of packets are lost), and the situation with $80 \%$ of lost packets. It means our model is efficient with few information. The packet loss has no significant impact on the waiting time ( $1 s$ for the maximum difference).

Then, we test a heavy traffic (8500 trips). The figure 11 (B) shows results, we do not represent $80 \%$ of lost packet, because they are too bad. When the rate equals to 40 or $20 \%$, results are better than with all packets. The relation between the lost packets and the conflicts computed is represented in figure 
12. and we notice that when 40 or $20 \%$ of packets are lost, some conflicts are not solved and this avoids other conflicts and reduce the waiting time and travel time, however when too many packets are lost, too many conflicts are not solved and performance decreases. It means that it's not necessary to resolve all conflicts (only 60 or $80 \%$ are sufficient) in case of a great quality of the channel, therefore the proba in the algorithm can be fixed at 60 or $80 \%$. In case of poor quality, the proba has to be equal to $100 \%$ in order to be resistant to a packet loss of 40 or $20 \%$.
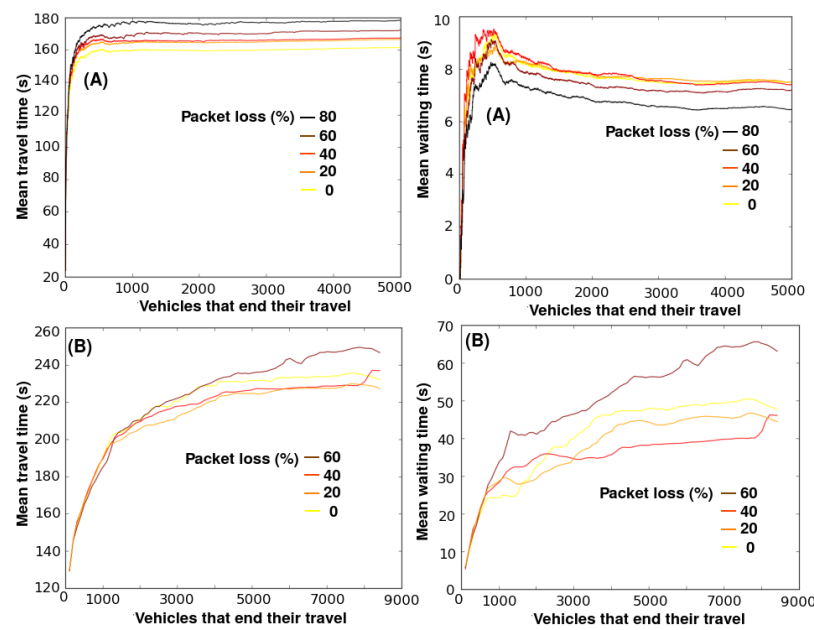

Fig. 11: Mean travel time and waiting time with different rate of packet loss with : (A) real traffic, (B) heavy traffic

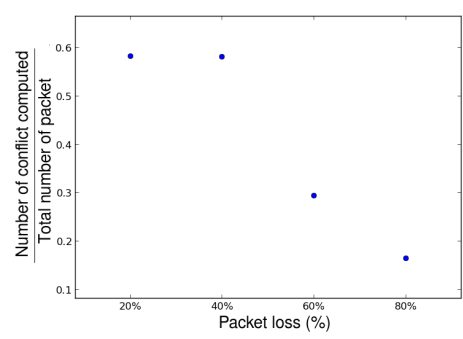

Fig. 12: Relation between the packet loss rate and conflicts solved

\section{F. Robustness: Radius of communication}

To complete our study, we evaluate the impact of five radius of communication. Vehicles start to receive and send data at 20,50,100,200,300 meters from the roundabout in each direction $(\mathrm{N}, \mathrm{S}, \mathrm{E}, \mathrm{W})$. Then we test a radius in function of the length of the incoming roads, 100 meters for South road, 90 meters for North road, and 200 meters for East and West roads. The results for the last simulations are called According to the length of the road in figure 13. The radius has no impact on the waiting time, however for the travel time a radius according the length of the road improves the total travel time to $10 \mathrm{~s}$ in case of real traffic.

Then we test with a heavy traffic (8500 trips). The figure 13 (B) shows that hort ranges of communication $(20,50,100$ meters) have a significant impact in the results in case of heavy traffic. Vehicles are not able to react when they receive informations too close to the intersection and conflicts can't be resolved. However with a distance of 200 meters but also according the length of the roads, results are constant and better than short ranges. We must note that the travel time and the waiting time, decrease at the end of the simulation in case of congestion, because the arrival law is null.
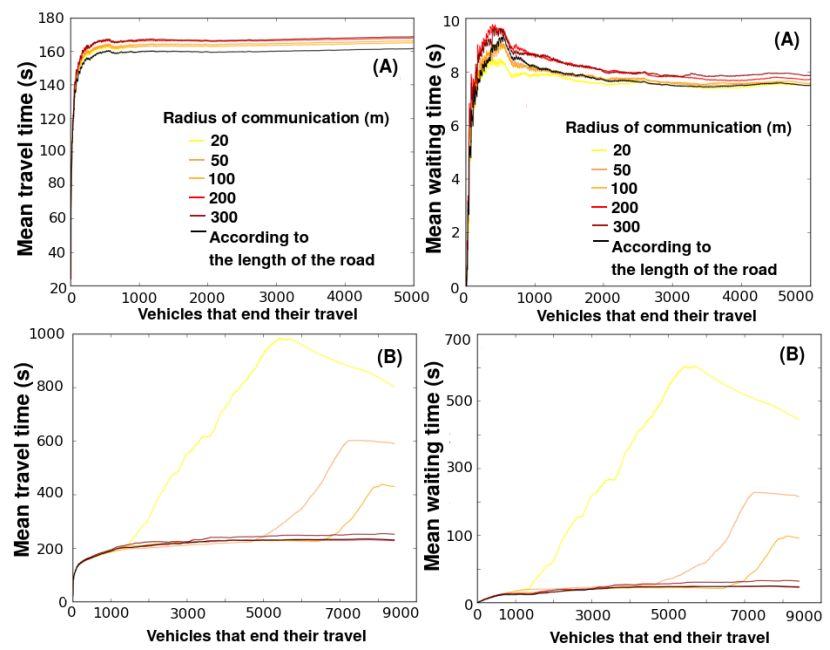

Fig. 13: Mean travel time and waiting time with different radius of communication with: (A) real traffic, (B) heavy traffic

\section{CONCLUSION}

This paper presents a decentralized V2V model which eliminates the potential overlaps of vehicular trajectories coming from all opposing directions. The system is able to control the traffic individually without a centralized infrastructure. It's efficient with only $50 \%$ of equipment and is resistant to packet loss. The model is probabilistic: in case of heavy traffic, by solving 60 to $80 \%$ of conflicts -or with 20 to $40 \%$ of packet lost-, our model performs good results in waiting time and travel time. Our paper demonstrates the potential of a decentralized communication between vehicles with a simple model on a complex roundabout. In the future we will provide more validation in different environments. REFERENCES

[1] Z. Li, M. Chitturi, D. Zheng, A. Bill, and D. Noyce, "Modeling reservation-based autonomous intersection control in vissim," Journal of the Transportation Research Board, no. 2381, pp. 81-90, 2013.

[2] M. Ahmane, A. Abbas-Turki, F. Perronnet, J. Wu, A. El Moudni, J. Buisson, and R. Zeo, "Modeling and controlling an isolated urban intersection based on cooperative vehicles," Transportation Research Part C: Emerging Technologies, vol. 28, pp. 44-62, 2013.

[3] T.-C. Au and P. Stone, "Motion planning algorithms for autonomous intersection management," in $A A A I, 2010$.

[4] J. Alonso, V. Milanés, J. Pérez, E. Onieva, C. González, and T. de Pedro, "Autonomous vehicle control systems for safe crossroads," Transportation Research Part C, vol. 19, no. 6, pp. 1095-1110, 2011.

[5] G. R. de Campos, P. Falcone, and J. Sjoberg, "Autonomous cooperative driving: a velocity-based negotiation approach for intersection crossing," in IEEE 16th ITSC, 2013, pp. 1456-1461.

[6] M.-A. Lèbre, F. Le Mouël, and E. Ménard, "Microscopic vehicular mobility trace of europarc roundabout, creteil, france," 2015.

[7] J. Cheng, X. Yang, W. Deng, and X. Huang, "Driver's critical gap calibration at urban roundabouts: a case study in china," Tsinghua Science and Technology, vol. 13, no. 2, pp. 237-242, 2008.

[8] S. Fujii, A. Fujita, T. Umedu, S. Kaneda, H. Yamaguchi, T. Higashino, and M. Takai, "Cooperative vehicle positioning via V2V communications and onboard sensors," in IEEE VTC, 2011.

[9] Q. Jin, G. Wu, K. Boriboonsomsin, and M. Barth, "Multi-agent intersection management for connected vehicles using an optimal scheduling approach," in ICCVE. IEEE, 2012, pp. 185-190.

[10] J. Wu, A. Abbas-Turki, and A. El Moudni, "Contextualized traffic controlling at isolated urban intersection," in The 14th WMSCI, 2010. 